\title{
Role of the nitric oxide metabolic pathway and prostanoids in the pathogenesis of endothelial dysfunction and essential hypertension in young men
}

\author{
Adrian Doroszko, Ryszard Andrzejak and Andrzej Szuba
}

The aim of this study was to explore the role of selected prostanoids and the nitric oxide (NO) metabolic pathway in the pathogenesis of endothelial dysfunction (ED) in young men with and without essential hypertension (HTN). A total of 70 men aged 18-40 years old (23 hypertensive and 47 normotensive) were investigated. Initial metabolite concentrations of the NO pathway (asymmetric dimethylarginine, L-arginine and symmetric dimethylarginine), selected cardiovascular risk markers (serum lipids, creatinine, glucose and high-sensitivity $\mathrm{C}$-reactive protein), oxidative stress markers (malonylodialdehyde, thiol index and nitrotyrosine) and prostanoids (thromboxane B2 ( $\left.\mathrm{TxB}_{2}\right)$ and 6-keto-prostaglandin F (PGF)-1- $\alpha$ ) were measured. Ultrasound assessment of endothelial function (flow-mediated vasodilation (FMD)) of the brachial artery was studied before and after intravenous infusion of L-arginine (16.0 g). All measurements were repeated after oral administration of indomethacin ( $75 \mathrm{mg}$ per day) for 2 days. The prevalence of ED was similar in both hypertensive and normotensive groups. A lower baseline plasma level of 6-keto-PGF-1- $\alpha$ and a higher baseline of $\mathrm{TxB}_{2}$ were observed in the hypertensive group. A different response to indomethacin assessed by prostanoid levels was observed and was dependent on the presence of HTN. No significant differences in metabolites of the NO pathway were observed at either baseline or following indomethacin treatment. In hypertensive patients, L-arginine and indomethacin had a synergistic positive effect on FMD. ED in young normotensive men primarily depends on NO deficiency. In young hypertensive men, disorders in prostanoid metabolism have important roles in decreasing NO bioavailability. The high prevalence of ED in potentially healthy subjects suggests that the ultrasound FMD measurement is an important tool in the stratification of cardiovascular risk. Hypertension Research (2011) 34, 79-86; doi:10.1038/hr.2010.169; published online 7 October 2010

Keywords: endothelial dysfunction; nitric oxide; oxidative stress; prostanoids

\section{INTRODUCTION}

The endothelium has a pivotal role in the regulation of vascular tone. Endothelial dysfunction (ED) resulting from decreased activity of nitric oxide (NO)-dependent metabolic pathways, which may be a crucial pathophysiological link that leads to the development of cardiovascular complications of arterial hypertension (HTN). ${ }^{1}$ Studies in hypertensive subjects have shown that ED exists in resistance vessels and is manifested by a decreased response to vasodilatory agents. ${ }^{2-4} \mathrm{ED}$ may be detected in hypertensive subjects, as well as in healthy individuals with cardiovascular risk factors or in those genetically predisposed to developing arterial HTN. Furthermore, it has been shown that ED is an independent risk factor for future cardiovascular events. ${ }^{5-9}$

A deficiency of NO has complex etiology; one of the most important causes is enhanced NO degradation by reactive oxygen species (ROS). Impaired activity of NO-dependent metabolic pathways in the endothelium may constitute an important pathophysiological step in the development of cardiovascular disease and is considered to have a causative role in the impairment of flow-mediated vasodilation (FMD). ${ }^{10,11}$
Asymmetric dimethylarginine (ADMA) is an endogenous competitive inhibitor of NO synthase (NOS) and may thus decrease NO bioavailability. ADMA is an important marker of cardiovascular risk $^{12,13}$ and may mediate ED in patients with coronary artery disease, arterial HTN, hyperlipidemia, congestive heart failure, ${ }^{14}$ chronic kidney disease $\mathrm{e}^{15}$ and/or cerebral stroke. ${ }^{16}$ ADMA and symmetric dimethylarginine (SDMA; without inhibitory action on NOS) are produced from methylated nuclear proteins, especially histones. ${ }^{13}$

Disorders involving the biotransformation of arachidonic acid derivates (prostanoids) are also postulated to be an important factor in ED pathogenesis. ${ }^{17}$ An imbalance between the vasoconstrictive action of some prostanoids (such as thromboxane) and the vasodilatory action of others (for example, prostacyclin) could also have an important role in the pathogenesis of HTN. ED may develop in normotensive subjects and might be eliminated by inhibition of cyclooxygenase (COX), which could be due to the decreased lipid peroxidation, resulting in a reduction in oxidative stress. ${ }^{18}$

Viridis et al. ${ }^{19}$ analyzed the role of the decreased availability of NO and ROS and activity of each isoform of COX in the development of 
ED. In addition, rats exposed to an endotoxin had a significant impairment in endothelial function, which was rescued after treatment with a COX-2 inhibitor. The authors suggested that NO was scavenged by ROS produced via the COX-2 pathway. Regardless of enhanced NOS activity, a decrease of NO bioavailability is explained by the coincident generation of ROS, which transforms NO into peroxynitrites $\left(\mathrm{ONOO}^{-}\right)$. Formation of ONOO- in vivo has been attributed to the reaction between the free radicals superoxide ( $\left.\mathrm{O}_{2}-\right)$ and $\mathrm{NO}$ (reviewed in Pacher et al. ${ }^{20}$ ). Nitrotyrosine formation is an $\mathrm{ONOO}^{-}$dependent mechanism, and its level is commonly used as a marker of $\mathrm{ONOO}^{-}$production.

HTN might be associated with altered expression in the $\mathrm{NO}$ and prostanoids pathways, ${ }^{21}$ as early as 30 years of age; however, the role of prostanoids appears to increase with age. ${ }^{22}$ Campia et al. ${ }^{23}$ performed a study in healthy and hypertensive subjects with or without lipid disorders and demonstrated a crucial role of prostanoids in the regulation of vascular tone. Vasoconstrictive prostanoids could also limit endothelium-mediated vasodilatation. The deficiency of NO might be more common in young subjects (under the age of 30 years) and may be reversible by administration of L-arginine ${ }^{24}$; however, in elderly subjects (over 60), ${ }^{25}$ disturbances of the prostanoid pathway might be more important. ${ }^{25}$ The results of a study performed by Bode-Boger ${ }^{26}$ however, oppose this hypothesis by showing a significant improvement of endothelial function in elderly subjects after administration of L-arginine.

The aim of this study was to assess the role of NO and prostanoids in the pathogenesis of ED in young men with and without HTN. We investigated (1) whether decreased bioavailability of NO in subjects with ED results from its enhanced degradation by ROS produced during lipid peroxidation or by its decreased synthesis due to competitive inhibition of NOS; (2) if administration of L-arginine (a substrate for NOS) increases NO bioavailability (that is, improving FMD) by enhancing its synthesis, which is inhibited by competitive NOS inhibitors; (3) whether administration of indomethacin (a non-selective COX inhibitor) reduces peroxidation of lipids, resulting in an increase of NO bioavailability and (4) if there are any differences between hypertensive and normotensive subjects in the pathogenesis of ED.

\section{METHODS}

All experiments were conducted and approved in accordance with the guidelines of the Bioethics Committee at Wroclaw Medical University and adhered to the principles of the Declaration of Helsinki and Title 45, US Code of Federal Regulations, Part 46, Protection of Human Subjects (revised 13 November 2001, effective 13 December 2001).

\section{Patients}

Male volunteers (mean age, 18-40 years) from the university community in Wroclaw, Poland, were recruited to participate in this study from leaflets distributed in the campus area in Wroclaw. A total of 23 subjects with HTN and 47 normotensive subjects were included in the study. The recruited subjects were either healthy, normotensive individuals or had a history of untreated essential HTN for at least last 6 months. Exclusion criteria for the study were secondary HTN, coexisting with diabetes mellitus, chronic inflammatory diseases, infections, smoking, malignancies and mental disorders. Subjects with unknown histories of HTN and elevated blood pressure observed at two consecutive screening visits underwent a 24-h ambulatory blood pressure monitoring using a Welch Allyn ABPM 6100S (Welch Allyn Poland \& Baltic States, Poznan, Poland). If HTN was confirmed, ${ }^{27}$ subjects were given subsequent clinical evaluation to exclude secondary HTN. All blood pressure measurements were taken using a sphygmomanometer (Welch Allyn, DS66 Trigger Aneroid with a set of adult cuffs, size $10-12 \mathrm{~cm}$ ).

\section{Study protocol}

A scheme of the study protocol is shown in Figure 1a. Each subject was given a medical examination. At 06:30 hours on the day after the medical exam, fasting blood was drawn from the antecubital vein. To avoid platelet activation, blood was drawn without venous stasis. The following parameters were measured:

1. Metabolites of the NO pathway (ADMA, SDMA and L-arginine)

2. Prostanoids levels (thromboxane $\mathrm{B} 2\left(\mathrm{TxB}_{2}\right)$ and 6-keto-PGF-1- $\alpha$ )

3. Markers of oxidative stress (malondialdehyde (MDA), reduced glutathione (GSH), oxidized glutathione (GSSG), thiol index (GSH/GSSG) and nitrotyrosine)

4. Concentrations of total cholesterol, low-density lipoprotein and highdensity lipoprotein cholesterols, triglycerides, serum creatinine, plasma glucose, high-sensitivity C-reactive protein, thyroid-stimulating hormone, thyrotropin, $\mathrm{K}^{+}$and uric acid.

After the blood was drawn, the vasodilatory reactivity of the brachial artery after reversible ischemia (FMD) was measured to diagnose ED. The cutoff point for ED was established as $<10 \%$ change in the diameter of the brachial artery in response to reactive hyperemia compared with the baseline value. Subjects were assigned to one of four subgroups according to the presence of HTN and ED (Figure 1b):

(1) Normal blood pressure and no ED: $\operatorname{HTN}(-) / \operatorname{ED}(-)$

(2) Normal blood pressure but with ED: $\operatorname{HTN}(-) / \operatorname{ED}(+)$

(3) HTN without ED: $\operatorname{HTN}(+) / \operatorname{ED}(-)$

(4) HTN with ED: $\mathrm{HTN}(+) / \mathrm{ED}(+)$

Following the baseline FMD testing, the subjects were administered $16.0 \mathrm{~g}$ L-arginine (Fresenius SE, Homburg, Germany) intravenously, followed by a second FMD measurement.

After testing, all subjects were given $75 \mathrm{mg}$ of slow-release indomethacin per os. (Metindol Retard, Polfa Warszawa, Poland) daily for 2 days, after which all procedures were repeated.

\section{Procedure}

Measurements of endothelial function. Ultrasound assessment of FMD in response to reactive hyperemia was performed according to the method described by Celermajer et al. ${ }^{28}$ using the ALOKA model SSD 5500 duplex ultrasound machine (ALOKA GmbH, Willich, Germany) with a 7-14-MHz linear array transducer and the ALOKA probe holder MP-AH 0001 for immobilization of the arm/forearm. This was done to improve the accuracy of the FMD measurement.

Biochemical tests. EDTA plasma $(2.7 \mathrm{ml})$ was separated, immediately centrifuged (6000 r.p.m. for $15 \mathrm{~min}$ at $4{ }^{\circ} \mathrm{C}$ ) and frozen at $-80^{\circ} \mathrm{C}$ for evaluation of ADMA, SDMA, L-arginine levels and markers of oxidative stress. Whole blood was used only for assessment of the thiol index, as recommended. Blood for assessment of prostanoids levels was drawn $(\mathrm{v}=2.7 \mathrm{ml})$ into sodium citrate tubes with $500 \mu \mathrm{l}$ of $5 \mathrm{mmoll}^{-1}$ crystallic acetylsalicylic acid (Sigma-Aldrich Sp. z o.o., Poznań, Poland) in $0.9 \%$ saline solution to inhibit ex vivo platelet activity. Blood was immediately centrifuged ( $6000 \mathrm{r.p.m}$. for $15 \mathrm{~min}$ at $4^{\circ} \mathrm{C}$ ), and plasma was separated and stored at $-80^{\circ} \mathrm{C}$ until analysis. The blood for the remaining biochemical analyses was allowed to clot. It was then centrifuged for subsequent analyses.

Assessment of ADMA, SDMA and L-arginine levels. Plasma concentrations of L-arginine, $N^{\mathrm{G}}, N^{\mathrm{G}^{\prime}}$-dimethylarginine (ADMA) and $N^{\mathrm{G}}, N^{\mathrm{G}}$-dimethylarginine (SDMA) were measured by high-performance liquid chromatography, and precolumn derivatization was measured with $o$-phthaldialdehyde by a previously published method. ${ }^{13}$

Markers of oxidative stress. MDA levels were assessed using a lipoperoxidation marker using a colorimetric assay (LPO-586, BIOXYTECH, OXIS International, Inc., Beverly Hills, CA, USA). In this method, a reaction of $\mathrm{N}$-methyl-O-2-phenylindole with MDA is used and results in the synthesis of a chromogenic product $\left(\lambda_{\max }=586 \mathrm{~nm}\right)$. Addition of $\mathrm{HCl}$ inhibits crossreactivity for hydroxyalkenal; thus, the results reflect only the level of MDA..$^{29,30}$

The thiol index (GSH/GSSG), the ratio of reduced to oxidated glutathione, was measured using a colorimetric assay (GSH/GSSG-412 BIOXYTECH) described by Gunthenberg. ${ }^{31}$ 
a



Figure 1 (a) Scheme of study protocol. First, medical examination of each subject was performed, followed by blood pressure measurement. Next, blood samples for subsequent analyses were drawn, and a baseline flow-mediated vasodilation (FMD) measurement was performed. Subsequently, 16.0 g of L-arginine was administered intravenously, followed by a second ultrasound FMD measurement. Each subject was then told to take an oral slow-release indomethacin tablet ( $75 \mathrm{mg}$ per day for 2 days). On the day 3, the protocol was repeated. (b) Schematic of the subject categorization according to the values of blood pressure (normotensive vs. hypertensive) and FMD of the brachial artery (with endothelial dysfunction (ED) vs. without ED).

Peroxynitrite production was determined by measuring nitrotyrosine levels using an enzyme immunoassay for nitrotyrosine (BIOXYTECH). ${ }^{32,33}$

Measurement of prostanoids level. Plasma concentrations of prostanoids (6-keto-PGF-1- $\alpha$ as a marker of prostacyclin synthesis and $\mathrm{TxB}_{2}$ reflecting thromboxane formation) were measured using commercial ELISA kits (Assay Designs-EIA kit 6-keto-PGF-1-a and TxB2 enzyme immunoassay EIA kit; BIOMIBO, Warszawa, Poland).

Other biochemical analyses. Concentrations of total cholesterol, low-density lipoprotein and high-density lipoprotein fractions and triglycerides, serum creatinine, fasting plasma glucose, high-sensitivity C-reactive protein, thyroidstimulating hormone, thyrotropin, $\mathrm{K}^{+}$and uric acid were measured by standard laboratory assays. Serum insulin levels were measured with a commercial AxSYM Insulin Assay (Abbott Laboratories Poland Sp. z o.o., Warszawa, Poland) based on the Microparticle Enzyme Immunoassay (MEIA) technology. The formulas used to calculate the homeostasis model assessment for insulin resistance (HOMA-IR) and to calculate the homeostasis model assessments for insulin secretion (HOMA- $\beta$ ) were

1. HOMA IR $=\left[\right.$ fasting insulin $\left(\mu \mathrm{U} \mathrm{ml}^{-1}\right) \times$ fasting glucose $\left.\left(\mathrm{mmoll}^{-1}\right)\right] / 22.5$

2. HOMA- $\beta=\left[20 \times\left(\right.\right.$ fasting insulin $\left.\left(\mu \mathrm{Uml}^{-1}\right)\right) /\left(\right.$ fasting glucose $\left(\mathrm{mmoll}^{-1}\right)$ 3.5)].

Statistical analysis. Data are expressed as the mean \pm s.e.m. The differences between two continuous parameters were assessed using a Mann-Whitney 
$U$-test or a Student's $t$-test, followed by a Shapiro-Wilk test and Levene's test as appropriate. For comparison of more than two groups, an ANOVA followed by Tukey's test or a Kruskal-Wallis test (for non-parametric statistics) was performed. Correlations were assessed with a Pearson Moment or Spearman's test as appropriate. All calculations were performed using Statistica 6.0 StatSoft (StatSoft Poland Sp. z o.o., Kraków, Poland).

\section{RESULTS}

Blood pressure

Blood pressure was significantly higher in hypertensive subjects $(n=23)$ compared with those who were normotensive $(n=47$; systolic blood pressure $=148.1 \mathrm{vs} .124 .5 \mathrm{~mm} \mathrm{Hg}$, diastolic blood pressure $=91.4$ vs. $78.3 \mathrm{~mm} \mathrm{Hg}$, mean arterial pressure $=110.3$ vs. $93.7 \mathrm{~mm} \mathrm{Hg}$, pulse pressure $=56.7$ vs. $46.2 \mathrm{~mm} \mathrm{Hg}$, respectively; $P<0.05$ ).

\section{Baseline characteristics of investigated population}

All four subgroups had very similar demographic characteristics. There were no significant differences in the basic biochemistry between analyzed subgroups or in other markers of cardiovascular risk (Table 1). Insulin levels and HOMA-IR values were non-significantly higher in hypertensive subjects, and HOMA- $\beta$ was non-significantly lower in subgroups with ED (Table 1). None of the recruited patients were treated with any drugs, and none suffered from any chronic disease.

Endothelial function at baseline and after administration of L-arginine and indomethacin

There were no significant differences in FMD of the brachial artery at baseline between hypertensive and normotensive subjects. There was, however, a significant increase in FMD values in response to intravenous infusion of L-arginine in both subgroups with $\mathrm{ED}$, independent of the presence of HTN (Figure 2a). Administration of indomethacin resulted in an improvement of endothelial function in subgroups with ED. Infusion of L-arginine after pretreatment with indomethacin resulted in further improvement of endothelial function, but only in hypertensive subjects, even in those with normal FMD values at baseline. No significant changes in FMD values in subgroups without HTN (compared with the values before L-arginine infusion and after indomethacin treatment) were observed (Figure 2a).

\section{ADMA, SDMA and L-arginine levels}

There were no significant differences in baseline levels of ADMA, SDMA, L-arginine or L-arginine/ADMA ratio in the hypertensive $v s$. normotensive groups $\left(\mathrm{ADMA}=0.43 \pm 0.01\right.$ vs. $0.40 \pm 0.010 \mu \mathrm{moll}^{-1}$, SDMA $=0.22 \pm 0.01$ vs. $0.23 \pm 0.01 \mu \mathrm{moll}^{-1}$, $\mathrm{L}$-arginine $=35.8 \pm 3.7 v s$. $33.2 \pm 1.9 \mu \mathrm{moll}^{-1}$, respectively). No significant differences in the concentrations of these metabolites between all four investigated subgroups were observed.

\section{Markers of oxidative stress}

The baseline levels of MDA were similar in all subgroups and decreased after treatment with indomethacin (Figure 2b). A significantly lower baseline thiol index was observed in subgroups with ED compared with those with appropriate vasodilatory function of the endothelium. In subgroups with HTN, the thiol index was higher compared with those who were normotensive. Treatment with indomethacin led to an increase in the thiol index and a decrease in MDA levels in all subgroups, which was statistically significant only in subgroups with ED (Figure $2 \mathrm{~b}$ ). $\mathrm{ONOO}^{-}$production, as determined by nitrotyrosine levels, was significantly higher in subgroups with ED, independent of the presence of HTN. A significant decrease in nitrotyrosine levels after treatment with indomethacin was observed only in the subgroup with ED and HTN (Figure 2c).

Table 1 Demographic characteristics of investigated population divided according to presence of hypertension (HTN) and endothelial dysfunction (ED)

\begin{tabular}{|c|c|c|c|c|c|}
\hline Parameter & $\begin{array}{c}E D(-) / H T N(-), \mathrm{n}=14 \\
(\text { mean } \pm \text { s.e.m. })\end{array}$ & $\begin{array}{c}E D(+) / H T N(-), \mathrm{n}=33 \\
(\text { mean } \pm \text { s.e.m. })\end{array}$ & $\begin{array}{c}E D(-) / H T N(+), \mathrm{n}=7 \\
(\text { mean } \pm \text { s.e.m. })\end{array}$ & $\begin{array}{c}E D(+) / H T N(+), \mathrm{n}=16 \\
(\text { mean } \pm \text { s.e.m. })\end{array}$ & P-value \\
\hline Age (years) & $25.5 \pm 1.21$ & $26.5 \pm 1.24$ & $23.3 \pm 2.1$ & $26.9 \pm 1.9$ & NS \\
\hline $\mathrm{BMI}\left(\mathrm{kg} \mathrm{m}^{-2}\right)$ & $25.0 \pm 0.52$ & $26.4 \pm 0.84$ & $26.5 \pm 1.7$ & $26.8 \pm 1.0$ & NS \\
\hline WHR (1) & $0.93 \pm 0.01$ & $0.92 \pm 0.02$ & $0.93 \pm 0.03$ & $0.96 \pm 0.01$ & NS \\
\hline $\mathrm{SBP}(\mathrm{mm} \mathrm{Hg})$ & $118.8 \pm 3.13$ & $126.8 \pm 1.93$ & $149.1 \pm 2.4^{*, \dagger}$ & $147.6 \pm 3.7^{*, \dagger}$ & $<0.05$ \\
\hline $\mathrm{DBP}(\mathrm{mm} \mathrm{Hg})$ & $77.1 \pm 1.99$ & $78.8 \pm 1.39$ & $93.3 \pm 2.4^{*, \dagger}$ & $90.7 \pm 2.2^{*, \dagger}$ & $<0.05$ \\
\hline MAP (mm Hg) & $91.0 \pm 2.2$ & $94.8 \pm 1.39$ & $111.9 \pm 2.3^{*, \dagger}$ & $109.7 \pm 2.4^{*, \dagger}$ & $<0.05$ \\
\hline $\mathrm{PP}(\mathrm{mm} \mathrm{Hg})$ & $41.8 \pm 2.3$ & $48.0 \pm 1.63$ & $55.8 \pm 2.0^{*, \dagger}$ & $57.0 \pm 3.0 *, \uparrow$ & $<0.05$ \\
\hline HR (b.p.m.) & $71.4 \pm 2.5$ & $76.6 \pm 2.35$ & $78.2 \pm 4.4$ & $74.6 \pm 2.1$ & NS \\
\hline hsCRP $\left(\mathrm{mg} \mathrm{l}^{-1}\right)$ & $1.83 \pm 1.03$ & $2.37 \pm 0.49$ & $2.04 \pm 0.22$ & $1.97 \pm 0.18$ & NS \\
\hline Fasting glucose $\left(\mathrm{mg} \mathrm{dl}^{-1}\right)$ & $88.4 \pm 3.98$ & $87.1 \pm 1.85$ & $86.4 \pm 3.5$ & $91.4 \pm 1.5$ & NS \\
\hline GFR $\left(\mathrm{ml} \mathrm{min}^{-1}\right)$ & $140.7 \pm 9.52$ & $140.7 \pm 4.25$ & $149.4 \pm 11.5$ & $141.1 \pm 6.2$ & NS \\
\hline Uric acid (mg dl-1) & $6.54 \pm 0.41$ & $6.14 \pm 0.24$ & $5.9 \pm 0.35$ & $5.9 \pm 0.4$ & NS \\
\hline $\mathrm{TCh}\left(\mathrm{mg} \mathrm{dl} \mathrm{l}^{-1}\right)$ & $177.2 \pm 10.3$ & $183.3 \pm 7.45$ & $172.1 \pm 10.5$ & $190.1 \pm 10.5$ & NS \\
\hline $\mathrm{TG}\left(\mathrm{mg} \mathrm{dl^{-1 }}\right)$ & $116.1 \pm 21.3$ & $110.5 \pm 9.8$ & $145.0 \pm 35.1$ & $137.6 \pm 18.7$ & NS \\
\hline $\mathrm{HDL}\left(\mathrm{mg} \mathrm{dl}^{-1}\right)$ & $50.1 \pm 3.31$ & $48.2 \pm 1.97$ & $49.5 \pm 3.1$ & $47.5 \pm 2.1$ & NS \\
\hline $\mathrm{LDL}\left(\mathrm{mg} \mathrm{dl}^{-1}\right)$ & $104.8 \pm 8.2$ & $116.9 \pm 6.9$ & $93.5 \pm 5.3$ & $110.1 \pm 6.6$ & NS \\
\hline $\mathrm{TSH}\left(\mathrm{mIU} \mathrm{I}^{-1}\right)$ & $2.55 \pm 0.38$ & $2.25 \pm 0.18$ & $2.82 \pm 0.6$ & $1.75 \pm 0.23$ & NS \\
\hline $\mathrm{K}^{+}\left(\mathrm{mEq} \mathrm{I}^{-1}\right)$ & $4.05 \pm 0.06$ & $4.15 \pm 0.08$ & $4.35 \pm 0.26$ & $4.23 \pm 0.10$ & NS \\
\hline Insulin $\left(\mu \cup \mathrm{ml}^{-1}\right)$ & $7.55 \pm 1.47$ & $8.16 \pm 0.71$ & $11.6 \pm 3.4$ & $13.2 \pm 3.2$ & NS \\
\hline HOMA-IR & $1.52 \pm 0.25$ & $1.82 \pm 0.17$ & $2.53 \pm 0.85$ & $2.32 \pm 0.45$ & NS \\
\hline HOMA- $\beta$ & $205.2 \pm 103.7$ & $108,9 \pm 15.1$ & $197.4 \pm 42.4$ & $140.6 \pm 31.5$ & NS \\
\hline
\end{tabular}

Abbreviations: BMI, body mass index; b.p.m, beats per minute; DBP, diastolic blood pressure; GFR, glomelural filtration rate; HDL, high-density lipoprotein; HOMA- $\beta$, homeostasis model assessments for insulin secretion; HOMA IR, homeostasis model assessment for insulin resistance; HR, heart rate; hsCRP, high-sensitivity C-reactive protein; LDL, low-density lipoprotein; MAP, mean arterial pressure; NS, not significant; PP, pulse pressure; SBP, systolic blood pressure; TCh, total cholesterol; TG, triglycerides; TSH, thyroid stimulating hormone; WHR, waist hips ratio. ${ }^{*} P<0.05$ vs. $\mathrm{ED}(-) / \mathrm{HTN}(-) ;{ }^{\dagger} P<0.05$ vs. $\mathrm{ED}(+) / \mathrm{HTN}(-)$. 


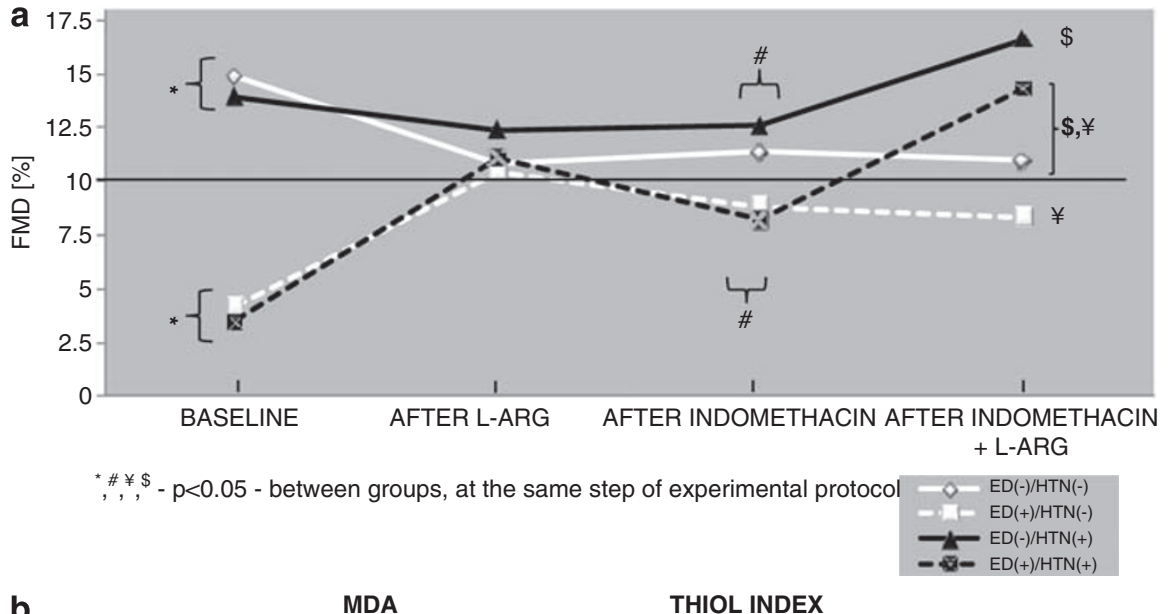

b

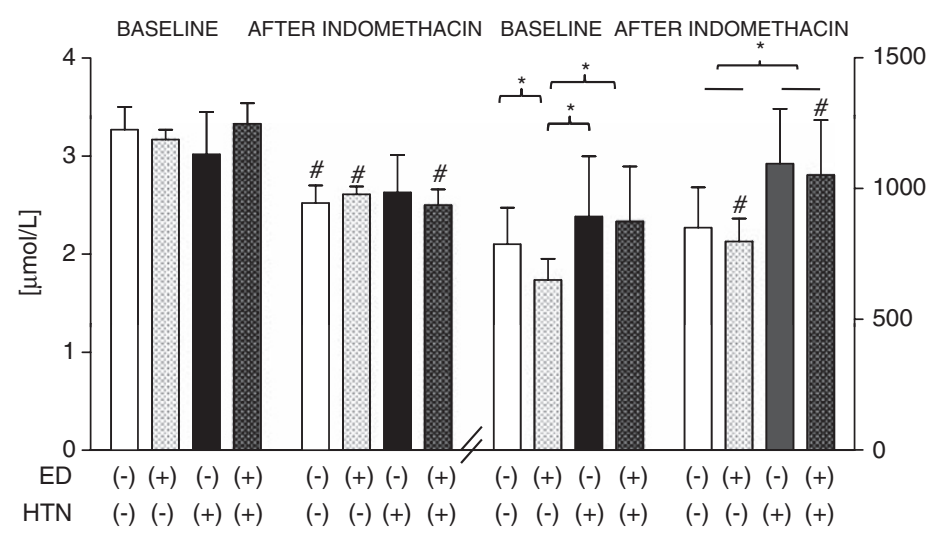

${ }^{*} \mathrm{p}<0.05$ between subgroups

$\# p<0.05$ vs. the same subgroup at baseline

C

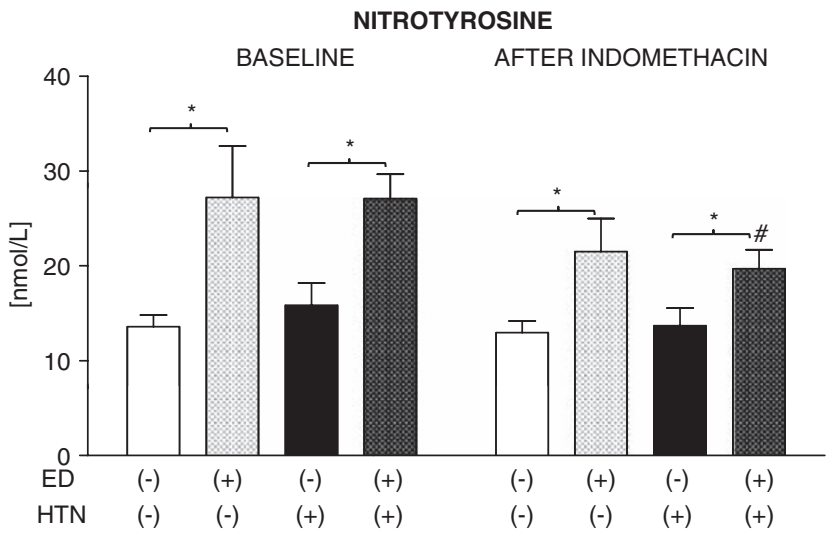

${ }^{*} \mathrm{p}<0.05$ between subgroups

$\# p<0.05$ vs. the same subgroup at baseline

Figure 2 (a) Characteristics of changes in vascular reactivity (flow-mediated vasodilation) in response to. L-arginine and/or indomethacin in the following subgroups: (1). ED(-) and HTN(-), $n=14$. (2). ED(+) and HTN(-), $n=33$. (3). ED(-) and HTN(+), $n=7$. (4). ED(+) and HTN(+), $n=14 .{ }^{*}, \#, \Psi, \$ P<0.05$ between groups, at the same step of experimental protocol. (b) Markers of oxidative stress at baseline and after administration of indomethacin. Means were compared between the four subgroups separated according to endothelial function and blood pressure at each step of the protocol. ${ }^{*} P<0.05$ between subgroups; ${ }^{\#} P<0.05$ vs. the same subgroup at baseline. (c) Comparison of nitrotyrosine levels and its changes between subgroups after treatment with indomethacin. ${ }^{*} P<0.05$ between subgroups; ${ }^{\#} P<0.05$ vs. the same subgroup at baseline. ED, endothelial dysfunction; HTN, hypertension; MDA, malonyldialdehyde; Thiol index, reduced glutathione (GSH)/oxidized glutathione (GSSG).

\section{Prostanoids}

The hypertensive group was characterized by a significantly lower plasma concentration of prostacyclin (6-keto-PGF-1- $\alpha$ levels: $534.6 \pm 78.6 \mathrm{ngl}^{-1}$ vs. $\left.1043.6 \pm 126.1 \mathrm{ngl}^{-1} \quad P<0.05\right)$ and a slightly higher baseline plasma level of $\mathrm{TxB}_{2} \quad\left(584.7 \pm 68.6 \mathrm{ngl}^{-1}\right.$ vs. $561.8 \pm 38.5 \mathrm{ngl}^{-1}$, without statistical significance). Furthermore, a significant increase in plasma prostacyclin levels was observed in subjects with HTN in response to indomethacin. Conversely, a 


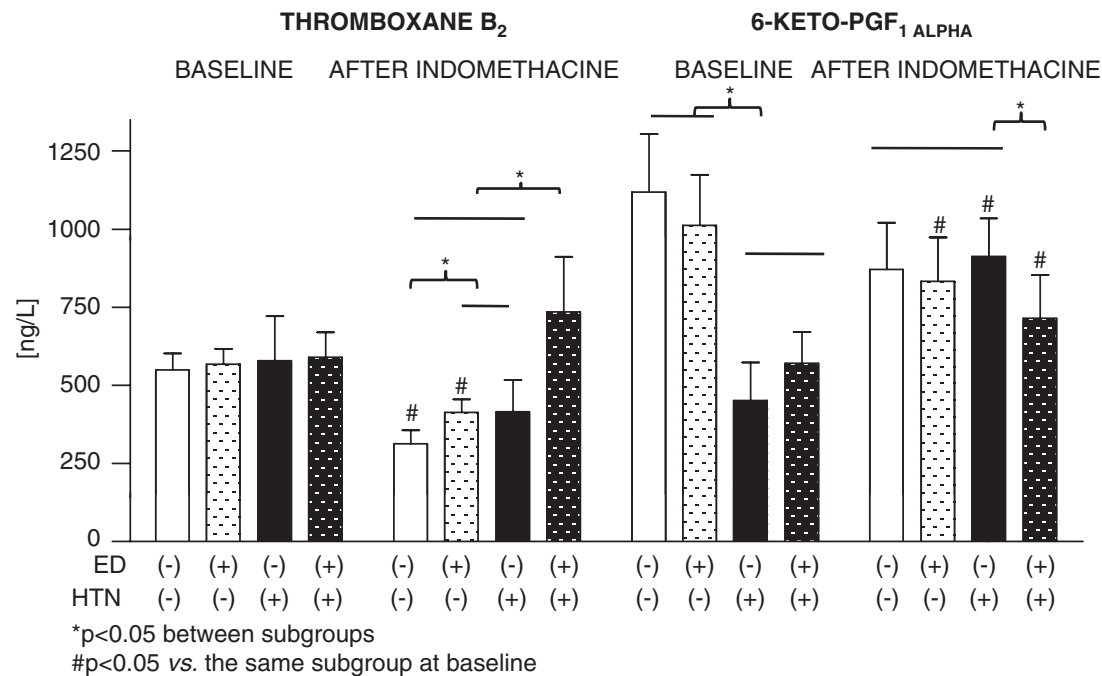

Figure 3 Prostanoids at baseline and after administration of indomethacin. Means were compared at the same step of the experimental protocol between the four subgroups separated according to endothelial function and blood pressure. ${ }^{*} P<0.05$ between subgroups; ${ }^{\#} P<0.05$ vs. the same subgroup at baseline. ED, endothelial dysfunction; HTN, hypertension.

decrease in plasma prostacyclin levels was observed in the normotensive group (Figure 3). Finally, thromboxane was decreased in normotensive subgroups after treatment with indomethacin (Figure 3).

\section{DISCUSSION}

To our knowledge, this is the first demonstration that vascular reactivity assessed by FMD is characterized by a different pattern of regulatory mechanisms in young men with essential HTN compared with normotensive subjects. The improvement of endothelial function in hypertensive subjects after L-arginine infusion was significantly potentiated by indomethacin pretreatment. Given that a blockage of the prostanoids pathway and the concurrent activation of $\mathrm{NO}$ synthesis are necessary to restore appropriate endothelial function, a cascade relationship between these two pathways is possible. Activation of the prostanoid pathway in hypertensive subjects may increase oxidative stress by increasing the formation of lipid peroxides, which subsequently scavenge NO. Therefore, administration of $\mathrm{L}$-arginine alone is not sufficient to restore appropriate FMD because of persistent NO scavenging. Inhibition of COX reduces peroxidation of lipids (as assessed by MDA measurements) and increases the serum anti-oxidative capacity (thiol index), resulting in increased NO bioavailability. Given that a synergistic improvement of FMD after administration of indomethacin is potentiated further by administration of $\mathrm{L}$-arginine in hypertensive subjects, a causative role of the increased oxidative stress in reducing $\mathrm{NO}$ bioavailability could be postulated in these subjects. A decrease in nitrotyrosine levels in these subjects after treatment with indomethacin confirms this hypothesis. Thus, our observation is consistent with earlier findings that a balance between NO and ROS is crucial for maintenance of vascular function. ${ }^{34,35}$ According to a study by Taddei et al., ${ }^{36}$ COX is responsible for ROS formation, which subsequently decreases NO bioavailability. Ge et al. ${ }^{37}$ postulated that HTN might be associated with higher COX-1 expression and that non-selective blockage of COX may result in lower synthesis of vasoconstrictive prostanoids. Long-term oxidative stress enhances COX-2 expression. This enhancement could be a reason for the enhanced synthesis of vasoconstrictive prostanoids and ROS, which actively decrease NO bioavailability.
This relationship, however, was not observed in normotensive subjects. Modulation of one pathway was demonstrated to be sufficient to restore FMD in the presence of ED. Moreover, in normotensive subjects, no synergy between the drugs was observed.

Contrary to other investigators, Schlaich et al. ${ }^{38}$ did not find significant differences in plasma levels of ADMA and L-arginine between healthy and hypertensive subjects. ${ }^{39}$ In our current study, we did not observe HTN-dependent differences in concentrations of ADMA, L-arginine or the L-arginine/ADMA ratio. Infusion of L-arginine resulted in a significant improvement of FMD in both groups with ED during the first day of the experiment, independent of the presence of HTN. Nevertheless, an inhibition of COX resulted in a further, synergistic improvement of FMD only in hypertensive subjects, indicating that oxidative stress is a primary mechanism of decreased NO bioavailability.

Takiuchi et al. ${ }^{40}$ showed that impaired vasodilatory function of coronary and peripheral vessels in subjects with HTN results from elevated plasma levels of ADMA. The results of our study are consistent with these findings, as we did not observe significant differences in ADMA plasma levels in young men, regardless of the presence of HTN. Thus, decreased NO bioavailability resulted from increased NO degradation, not from decreased NO synthesis (that is, increased ADMA levels).

Another novel finding in our study was the trend in the changes of thromboxane and prostacyclin levels after COX inhibition by indomethacin. An increase in 6-keto-PGF-1- $\alpha$ plasma levels was more common in hypertensive subjects; however, in normotensive subjects, a decrease in plasma PGI was more frequent. The observed increase in prostacyclin production despite treatment with nonsteroidal antiinflammatory drug could be explained by activation of the peroxisome proliferator-activated receptor (PPAR) $-\alpha$ and $-\gamma$ by some anti-inflammatory drugs. ${ }^{41,42}$ Given that the peroxisome proliferator-activated receptor response element is present within the promoter region of the COX-2 gene, increased expression of COX-2 might be responsible. Nevertheless, the presence of such regulation only in hypertensive subjects needs further investigation.

Independent of the presence of HTN, a similar prevalence of ED was observed. These findings suggest independent mechanisms that 
lead to the development of ED and HTN. ED is observed in subjects with HTN as well as in healthy subjects with increased cardiovascular risk. ${ }^{43}$ The phenotype of endothelial function, however, is different in hypertensive subjects compared with normotensive men. Administration of indomethacin potentiates response to L-arginine only in hypertensive subjects. This observation could indicate that HTN is associated with COX-dependent oxidative stress, which may lead to impairment of FMD due to increased NO scavenging. Elevated insulin levels and HOMA-IR values in hypertensive subjects could indicate an important role of metabolic disorders in the pathogenesis of HTN in young men. The increase in insulin levels and HOMA-IR values, however, did not reach statistical significance in our study. To verify this hypothesis, a larger population of subjects should be investigated.

To date, a 'normal range' of FMD values has not been established; therefore, the selection of the cut-off point is arbitrary and fluctuates from study to study (from 5 to 15\%). In one study performed on young healthy men, ${ }^{44}$ the mean FMD value was $9.7 \pm 1.4 \%$. In other studies, ${ }^{45,46}$ it has been shown that normal FMD values are higher in younger male subjects, and a regression model to assess the cut-off point for $\mathrm{ED}$ was calculated $[\mathrm{FMD}=25.5-(0.17 \times$ age $)-(2.6 \times$ resting vessel diameter)]. In this study, ED was recognized when the increase in FMD was $<10 \%$ of the increase in the diameter of the artery, which was likely because the investigated population included young men. Nevertheless, it is necessary to clearly establish a normal range of FMD values that takes into account gender and age.

\section{Limitations}

In this study, the vasodilation protocol following nitroglycerine was not investigated. As cardiovascular risk factors increase in number, smooth muscle dysfunction becomes apparent, and the nitroglycerin response is progressively impaired independently of ED. ${ }^{47}$ This should be taken into account when studying patients with either coronary or systemic atherosclerosis. ${ }^{48}$ Given that only young male subjects were investigated, we did not include administration of nitroglycerine in the study protocol.

\section{Perspectives}

The results of our study indicate different mechanisms underlying the regulation of vascular tone that are dependent on the presence of HTN. Moreover, the ED present in the two groups evaluated was shown to be triggered by different mechanisms. A divergent response to nonsteroidal anti-inflammatory drugs dependent on the presence of HTN may have important clinical implications, which will allow for the selection of patients for whom the treatment with non-steroidal anti-inflammatory/antiplatelet drugs or other strategies to reduce oxidative stress in the primary prevention of cardiovascular events will be beneficial. Hypertensive subjects with concomitant ED may constitute this group; however, further research is required to confirm this hypothesis. Given that the prevalence of ED in potentially healthy subjects is quite high, it might be reasonable to consider the assessment of FMD as an important tool in the stratification of cardiovascular risk in this group.

\section{CONFLICT OF INTEREST}

The authors declare no conflict of interest.

\section{ACKNOWLEDGEMENTS}

This project was founded from a grant subsidized by the Polish Ministry of Science and Higher Education (Grant No NN402259134). Study was supported by research fellowship within 'Development Program of Wroclaw Medical University' funded from European Social Fund, Human Capital, National Cohesion Strategy (contract no. UDA-POKL.04.01.01-00-010/08-00).
1 Nadar S, Blann AD, Lip GY. Endothelial dysfunction: methods of assessment and application to hypertension. Curr Pharm Des 2004; 10: 3591-3605.

2 Rizzoni D, Porteri E, Castellano M, Bettoni G, Muiesan ML, Tiberio GM, Giulini SM, Rossi G, Bernini G, Agabiti-Rosei E. Endothelial dysfunction in hypertension is independent from the etiology and from vascular structure. Hypertension 1998; 31: 335-341.

3 Luscher TF, Vanhoutte PM. Endothelium-dependent contractions to acetylcholine in the aorta of the spontaneously hypertensive rat. Hypertension 1986; 8: 344-348.

4 Yang D, Feletou M, Levens N, Zhang JN, Vanhoutte PM. A diffusible substance(s) mediates endothelium-dependent contractions to acetylcholine in the aorta of the spontaneously hypertensive rat. Hypertension 2003; 41: 143-148.

5 Chan SY, Mancini GB, Kuramoto L, Schultzer M, Frohlich J, Ignaszewski A. The prognostic importance of endothelial dysfunction and carotid atheroma burden in patients with coronary artery disease. J Am Coll Cardiol 2003; 42: 1037-1043.

6 Gokce N, Keaney Jr JF, Hunter LM, Nedeljkovic ZS, Menzoian JO, Vita JA. Predictive value of noninvasively determined endothelial dysfunction for long-term cardiovascular events in patients with peripheral vascular disease. J Am Coll Cardiol 2003; 41: 1769-1775.

7 Soga J, Nakamura S, Nishioka K, Umemura T, Jitsuiki D, Hidaka T, Teragawa $H$, Takemoto H, Goto C, Yoshizumi M, Chayama K, Higashi Y. Relationship between augmentation index and flow-mediated vasodilation in the brachial artery. Hypertens Res 2008; 31: 1293-1298.

8 Heitzer T, Baldus S, von Kodolitsch Y, Rudolph V, Meinertz T. Systemic endothelial dysfunction as an early predictor of adverse outcome in heart failure. Arterioscler Thromb Vasc Biol 2005; 25: 1174-1179.

9 Yufu K, Takahashi N, Hara M, Saikawa T, Yoshimatsu H. Measurement of the brachialankle pulse wave velocity and flow-mediated dilatation in young, healthy smokers. Hypertens Res 2007; 30: 607-612.

10 Taddei S, Virdis A, Ghiadoni L, Magagna A, Salvetti A. Vitamin C improves endotheliumdependent vasodilation by restoring NO activity in essential hypertension. Circulation 1998; 97: 2222-2229.

11 Heitzer T, Schlinzig T, Krohn K, Meinertz T, Munzel T. Endothelial dysfunction, oxidative stress, and risk of cardiovascular events in patients with coronary artery disease. Circulation 2001; 104: 2673-2678.

12 Chirinos JA, David R, Bralley JA, Zea-Díaz H, Muñoz-Atahualpa E, Corrales-Medina F, Cuba-Bustinza C, Chirinos-Pacheco J, Medina-Lezama J. Endogenous NOS inhibitors, arterial hemodynamics, and subclinical vascular disease: the PREVENCION Study. Hypertension 2008; 52: 1051-1059.

13 Böger RH, Bode-Böger SM, Szuba A, Tsao S, Chan JR, Tangphao O, Blaschke TF, Cooke JP. Asymmetric dimethylarginine (ADMA): a novel risk factor for endothelial dysfunction: its role in hypercholesterolemia. Circulation 1998; 98: 1842-1847.

14 Lavi S, Yang EH, Prasad A, Mathew V, Barsness GW, Rihal CS, Lerman LO, Lerman A. The interaction between coronary endothelial dysfunction, local oxidative stress, and endogenous NO in humans. Hypertension 2008; 51: 127-133.

15 Okubo K, Hayashi K, Wakino S, Matsuda H, Kubota E, Honda M, Tokuyama H, Yamamoto T, Kajiya F, Saruta T. Role of asymmetrical dimethylarginine in renal microvascular endothelial dysfunction in chronic renal failure with hypertension. Hypertens Res 2005; 28: 181-189.

16 Furuki K, Adachi H, Enomoto M, Otsuka M, Fukami A, Kumagae S, Matsuoka H, Nanjo Y, Kakuma T, Imaizumi T. Plasma level of asymmetric dimethylarginine (ADMA) as a predictor of carotid intima-media thickness progression: six-year prospective study using carotid ultrasonography. Hypertens Res 2008; 31: 1185-1189.

17 Widlansky M, Price D, Gokce N, Eberhardt RT, Duffy S, Holbrook M, Vita JA. Short- and long-term COX-2 inhibition reverses endothelial dysfunction in patients with hypertension. Hypertension 2003; 42: 310-315.

18 Blanco-Rivero J, Cachofeiro V, Lahera V, Aras-Lopez R, Marquez-Rodas I, Salaices M, Xavier FE, Ferrer M, Balfagon G. Participation of prostacyclin in endothelial dysfunction induced by aldosterone in normotensive and hypertensive rats. Hypertension 2005; 46 : 107-112.

19 Virdis A, Colucci R, Fornai M, Blandizzi C, Duranti E, Pinto S, Del Tacca M. Cyclooxygenase-2 inhibition improves vascular endothelial dysfunction in a rat model of endotoxic shock: role of iNOS and oxidative stress. J of Pharm and Exp Th 2005; 312: 945-953.

20 Pacher P, Beckman JS, Liaudet L. Nitric oxide and peroxynitrite in health and disease. Physiol rev 2007; 87: 315-424.

21 Virdis A, Colucci R, Versari D, Ghisu N, Fornai M, Antonioli L, Duranti E, Daghini E, Giannarelli C, Blandizzi C, Taddei S, Del Tacca M. Atorvastatin prevents endothelial dysfunction in mesenteric arteries from spontaneously hypertensive rats: role of cyclooxygenase 2-derived contracting prostanoids. Hypertension 2009; 5 : 1008-1016.

22 Bauersachs J, Schafer A. Endothelial dysfunction in heart failure: mechanisms and therapeutic approaches. Curr Vasc Pharmacol 2004; 2: 115-124.

23 Campia U, Choucair WK, Bryant MB, Quyyumi AA, Cardillo C, Panza JA. Role of cyclooxygenase products in the regulation of vascular tone and in the endothelial vasodilator function of normal, hypertensive, and hypercholesterolemic humans. Am J Cardiol 2002; 89: 286-290.

24 Cooke JP. Does ADMA cause endothelial dysfunction? Arterioscler Thromb Vasc Biol 2000; 20: 2032-2037.

25 Taddei S, Virdis A, Mattei P, Ghiadoni L, Basile-Fasolo C, Sudano I, Salvetti A. Hypertension causes premature aging of endothelial function in humans. Hypertension 1997; 29: 736-743. 
26 Bode-Boger SM, Muke J, Surdacki A, Brabant G, Boger RH, Frolich JC. Oral L-arginine improves endothelial function in healthy individuals older than 70 years. Vasc Med 2003; 8: 77-81.

27 Mancia G, De Backer G, Dominiczak A, Cifkova R, Fagard R, Germano G, Grassi G, Heagerty AM, Kjeldsen SE, Laurent S, Narkiewicz K, Ruilope L, Rynkiewicz A, Schmieder RE, Boudier HA, Zanchetti A. 2007 ESH-ESC Practice Guidelines for the Management of Arterial Hypertension: ESH-ESC Task Force on the Management of Arterial Hypertension. J Hypertens 2007; 25: 1751-1762.

28 Celermajer DS, Sorensen KE, Gooch VM, Spiegelhalter DJ, Miller OI, Sullivan ID, Lloyd JK, Deanfield JE. Non-invasive detection of endothelial dysfunction in children and adults at risk of atherosclerosis. Lancet 1992; 340: 1111-1115.

29 Esterbauer H, Schaur RJ, Zollner H. Chemistry and biochemistry of hydroxynonenal, malonyldialdehydeand related aldehydes. Free Rad Biol Med 1991; 11: 81-128.

30 Wendell PL. Measurement of oxidized glutathione and total glutathione in the perfused rat heart. Biochem J 1970; 117: 661-665.

31 Guntherberg H, Rost J. The true oxidized glutathione content of red blood cells obtained by new enzymatic and paper chromatographic methods. Anal Biochem 1966; 15: 205-210.

32 Kooy NW. Extensive tyrosine nitration in human myocardial inflammation: evidence for the presence of peroxynitrite. Crit Care Med 1997; 25: 812-819.

33 Kaur H, Halliwell B. Evidence for nitric-oxide-mediated oxidative damage in chronic inflammation: nitrotyrosine in serum and synovial fluid from rheumatoid patients. FEBS Lett 1994; 350: 9-12.

34 Kojda G, Harrison D. Interactions between NO and reactive oxygen species: pathophysiological importance in atherosclerosis, hypertension, diabetes and heart failure. Cardiovasc Res 1999; 43: 562-571.

35 Rodrigo R, Prat H, Passalacqua W, Araya J, Guichard C, Bächler JP. Relationship between oxidative stress and essential hypertension. Hypertens Res 2007; 30: 1159-1167.

36 Taddei S, Viridis A, Ghiadoni L, Salvetti G, Bernini G, Magagna A, Salvetti A. Agerelated reduction of $\mathrm{NO}$ availability and oxidative stress in humans. Hypertension 2001; 38: 274-279.

37 Ge T, Hughes H, Junquero DC, Wu KK, Vanhoutte PM, Boulanger CM. Endotheliumdependent contractions are associated with both augmented expression of prostaglan- din $\mathrm{H}$ synthase- 1 and hypersensitivity to prostaglandin $\mathrm{H} 2$ in the SHR aorta. Circ Res 1995; 76: 1003-1010.

38 Schlaich MP, Parnell M, Ahlers BA, Finch S, Marshall T, Zhang WZ, Kaye DM. Impaired L-Arginine transport and endothelial function in hypertensive and genetically predisposed normotensive subjects. Circulation 2004; 110: 3680-3686.

39 Tsikas D, Boger RH, Sandmann J, Bode-Boger SM, Frolich JC. Endogenous nitric oxide synthase inhibitors are responsible for the L-arginine paradox. FEBS Lett 2000; 478 : $1-3$.

40 Takiuchi S, Fujii H, Kamide K, Horio T, Nakatani S, Hiuge A, Rakugi H, Ogihara T, Kawano Y. Plasma asymmetric dimethylarginine and coronary and peripheral endothelial dysfunction in hypertensive patients. Am J Hypertens 2004; 17: 802-808.

41 Pang L, Nie M, Corbett L, Knox AJ. Cyclooxygenase-2 expression by nonsteroidal antiinflammatory drugs in human airway smooth muscle cells: role of peroxisome proliferator-activated receptors. J Immunol 2003; 170: 1043-1051.

42 Lehmann JM, Lenhard JM, Oliver BB, Ringold GM, Kliewer SA. Peroxisome proliferatoractivated receptors $\alpha$ and $\gamma$ are activated by indomethacin and other non-steroidal antiinflammatory drugs. J Biol Chem 1997; 272: 3406-3410.

43 Taddei S, Virdis A, Mattei P, Ghiadoni L, Gennari A, Basile Fasolo C, Sudano I, Salvetti A. Aging and endothelial function in normotensive subjects and essential hypertensive patients. Circulation 1995; 91: 1981-1987.

44 Uehata A, Lieberman EH, Gerhard MD, Anderson TJ, Ganz P, Polak JF, Creager MA, Yeung AC. Noninvasive assessment of endothelium-dependent flow-mediated dilation of the brachial artery. Vasc Med 1997; 2: 87-92.

45 Ryliškyte L, Ghiadoni L, Plantinga Y, Janaviciene S, Petrulioniene Z, Laucevicius A, Gintautas J. High-frequency ultrasonographic imaging of the endothelium-dependent flow-mediated dilation (FMD) in a brachial artery: normative ranges in a group on low CV risk subjects of different age groups. Proc West Pharmac Sc 2004; 47: 67-68.

$46 \mathrm{Kelm}$ M. Flow-mediated dilatation in human circulation: diagnostic and therapeutic aspects. Am J of Physiol Heart Circ 2002; 282: 1-5.

47 Moens AL, Goovaerts I, Claeys MJ, Vrints CJ. Flow-mediated vasodilation: a diagnostic instrument, or an experimental tool? Chest 2005; 127: 2254-2263.

48 Adams MR, Robinson J, McCredie R, Seale JP, Sorensen KE, Deanfield JE et al. Smooth muscle dysfunction occurs independently of impaired endothelium-dependent dilation in adults at risk of atherosclerosis. J Am Coll Cardiol 1998; 32: 123-127. 